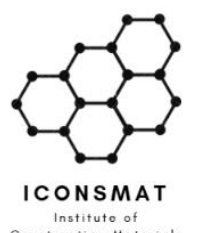

Institute of
Construction Materials
Content list available at ICONSMAT
Journal of Construction Materials
Journal homepage: www.iconsmat.com.au/publication

Article history:

Received 15 May 2020

Received in revised form

25 May 2020

Accepted 31 May 2020

Available online

2 July 2020

\title{
Diffusion of Innovation Theory in the Realm of Environmental Construction
}

\author{
Farid Sartipi ${ }^{1 *}$ \\ ${ }^{1}$ Institute of Construction Materials, NSW, Australia \\ *Corresponding author: Farid Sartipi, Researcher, Institute of Construction Materials, NSW, 2747, Australia. \\ E: farid.sartipi@iconsmat.com.au P: (+61) 0416731647
}

\begin{abstract}
Despite the enormous amount of investments that had been placed on the implementation of environmental construction practices, none of the proposed technologies had gained a widespread uptake. This lack of uptake appears to have roots somewhere apart from the engineering/technological aspect; more likely due to some behavioural characteristic. In this paper, the corresponding behavioural characteristics which are being considered as drawbacks towards the implementation of environmental practices will first be identified, and then based on each individual characteristic, effective measure will be outlined. The diffusion of innovation theory had been taken as the benchmark throughout the paper.
\end{abstract}

DOI: $10.36756 / J C M . v 1.3 .2$ (C2020 Institute of Construction Materials

\section{Keywords}

Innovation Theory; Built Environment; Behavioural Correctness 


\section{Introduction}

With increasing anthropogenic emissions in the industrial sector, individual sectors are encouraged to control their emission outputs by considering the relevant legislations. Construction industry is solely taking account for $50 \%$ of global greenhouse gas emission [1]. There had been many technological advancements developed along with this initiative to reduce the environmental impact of construction projects [2-4]. Life cycle assessment tools, as an example, failed to attract a widespread attention amongst the developers [5]. New material development, workflow modifications and many other promising attempts were so far deemed unsuccessful. It appears to be an inertia resisting the implementation such vital environmental practices.

There are certain behaviours that had been characterized in the construction business that are ingrained in the supply chain. One of them, just as an example, is the relatively high safety factors which are taught to help the developers in minimizing the risk of structural failure. Although essential, this approach usually leads to a higher consumption of materials which itself encourages the idea of "the thicker; the better". Thus, the modern construction industry needs to decharacterize some of the bad behaviours ingrained in the supply chain by using the power of institutions which is described by DiMaggio \& Powell's institutional theory [6] combined with the famous diffusion of innovation theory.

The main question that this article seeks an answer for is:

How can the establishment of the institutional pressures exerted within the construction supply chain aid the diffusion of low carbon construction innovation?

The culture of an industry is known as the factor influencing the behaviours within an industry. There are other similar phrases amongst the institutions which in fact deliver the same meaning such as, ethics guideline. This is in the power of policy makers to ensure compliance with the basic ethical guidelines i.e. the same power can change the direction of a system towards a more desirable ultimate [7]. In the context of construction industry, it is a good argument to state that any type of change requires a proper understanding of status quo. In other words, if the aim of the current paper is to establish an effective institutional pressure, then the typical behaviours ingrained within the industry must be outlined first. Values (which form the culture) within an organization are derived from various aspect. A company, for instance, may value customers above employees while another company vice versa [8]. In the pursuit of highly prioritize values in a social construct, one may also refer to the governmental foundations such as capitalism and socialism as well [9].

\section{DiMaggio \& Powell's institutional theory}

Institutional environment validate the outline strategies [10]. This is the subject in which DiMaggio \& Powell's institutional theory seeks to find the truth for. They argue that most of the discussions 
in the context of modern organizational structuration focus on differentiation, variation and diversity in behavioural characteristics. In their theory, however, homogeneity stands on top of variation. In fact, small business once they start their operation usually compete against each other in order to attract the market attention. However, in the later stages of life cycle, the tendency for convergence rises and that is the totality, unity to achieve higher goals, in later events which drives the organization. Getting back to the term "outlined organizational strategies", long term objectives, in this theory, are in pursuit of homogeneity which contains diversity, differentiation, and variation as crucial internal factors.

Isomorphism as another important terminology used in this theory refers to the set of interactions between the individuals/companies. The fundamental elements of an institutional organization respond the environment subject to the existing conditions. The same set of conditions then, as a result of the response from the organization will eventually change. And, that is the ethical constraints which put boundaries around the environment. This theory maintains the two types of isomorphism: competitive and institutional. In DiMaggio \& Powell's view point, competitive isomorphism, which sets the market competition as the core value, is not adequate for the modern organizations and needs to be supplemented by institutional isomorphism. In other words, the competitive isomorphism is meaningless without institutional isomorphism whilst institutional isomorphism is sufficient on its own. It is intrinsic in its definition that companies do not only compete for customers and economic wealth, but political and social power and legitimacy in order to remain at the top of their industry. One another relevant field of study in this area refers to the principles of cooperative and competitive game theory which is a mathematical investigation into the same concept [11]. DiMaggio and Powell's Institutional Theory establishes three types of isomorphism: coercive, mimetic, and normative (table 1).

TABLE 1 ISOMORPHIC PRESSURES AND DEFINITIONS

\section{Isomorphic pressure Definition}

Coercive

Coercive isomorphism derives from power and influence. In addition to this is can also arise from formal and informal pressures exerted by organisations on other companies upon which they are dependent. It also occurs sometimes due to cultural pressures within an industry.

Mimetic Mimetic isomorphism works on the basis of companies imitating each other. This occurs generally during times of uncertainty.

Normative Normative isomorphism occurs when the informal social rules of an industry have influence over decisions. 


\section{Diffusion of Innovation Theory}

This theory had been discussed first time by French sociologist Gabriel Tarde in 1903 and popularized by Everett Rogers in 1971. It argues that invention or innovation is not solely dependent on the technological competency itself but also the collaborative process. The perception of a new technological advancement is reliant on the characteristic and social class of the adopters. It urges the inventor to consider the target audience based on their current need and market their new product based on the classification proposed in this theory. In simple words, the innovator, in order to succeed in his commercialization process, needs to understand the personal characteristics of his audience. The significance of this theory become apparent once focus is placed on the collaborative pathway as a way of diffusion in such a fragmented supply chain within the construction industry. Secondly, the consumer driven approach in this theory is highly applicable in the construction industry as the client appears to be the most important actor in the supply chain. There are 5 distinct characterization for the adopters: Innovator, Early adopter, Early majority, Late majority, Laggards. Rogers demonstrated the proportion of each category in a quantified measured shown in the bell curve below.

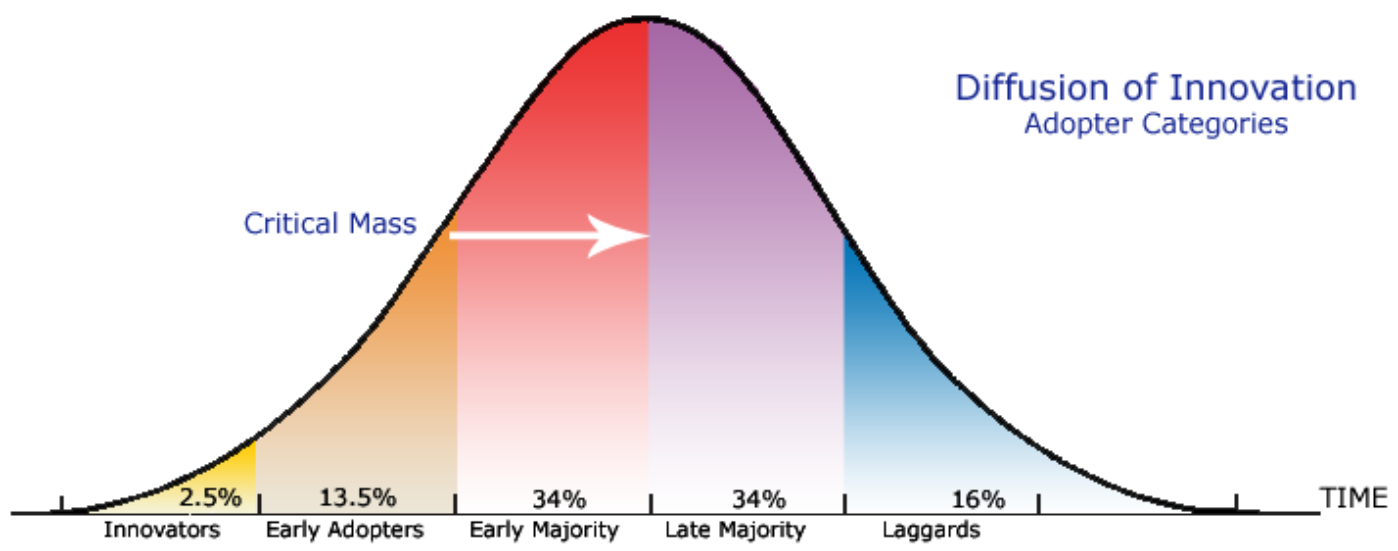

FIGURE 1 CATEGORIES OF ADOPTERS AND THEIR RELATIVE POPULATION

In this categorization, innovators are distinguished as high risk takers who have diverse social relationships and also significant financial backing. Early adopters are highly integrated into social systems and most likely to be consulted by potential innovation adopters. Early majority is the group of people who rely on informal information and usually take longer to adopt innovations. Late majority, on the other side, are sceptical and being attributed as cautious. For this group of people acceptance of peers is vital to their adoption. The fifth category of adopters are called laggards. They are slow to adopt, have strong inertia towards any change, poorly financed and traditional.

Each of these characteristics experience the adoption process as shown in figure 1 in their own unique way and by their own perception. The first step in the adoption process is where the data 
entry happens in the adopters' mind. This step is highly dependent on the instant psychological mood of the adopter which makes the enforcement process difficult with a high level of uncertainty. Nevertheless, repetition and persistent in the data entry is the most important part of this process i.e. deliver the same simple message multiple times until the hook catches the attention. In the second stage, after the initial acceptance, the adopter requires more information. This is now the art of the innovator to cautiously feed the adopter by the targeted set of information based on the characteristics of the adopter. One may ask, how to identify the individual's characteristics? And the answer to that relies on the predefined questionnaires which help in the assessment process. In the third stage of adoption process, the adopter after receiving enough information attempts to evaluate and analyze the options available to him. The adopter at this stage usually conduct a cost/benefit analysis and depending on their level of proficiency they might even dig into more details throughout a research process e.g. innovator's background, time dependent variables, etc. In the fourth stage of the adoption process, the adopter actually starts experiencing the invention/product. This is the step in which the ease of use and the level of the functionality of the new product will go under assessment. The famous term, "customer service" is in fact refers to the care matters and supports provided by the supplier. The adopters' feedback is largely important at this stage since a successful user experience leads the spread of the innovation through the so-called word-of-mouth which is known as the most effective way of marketing. In the final stage of adoption, based on the user experience in the

Knowledge or Awareness Stage:

Individual is exposed to innovation but lacks complete information

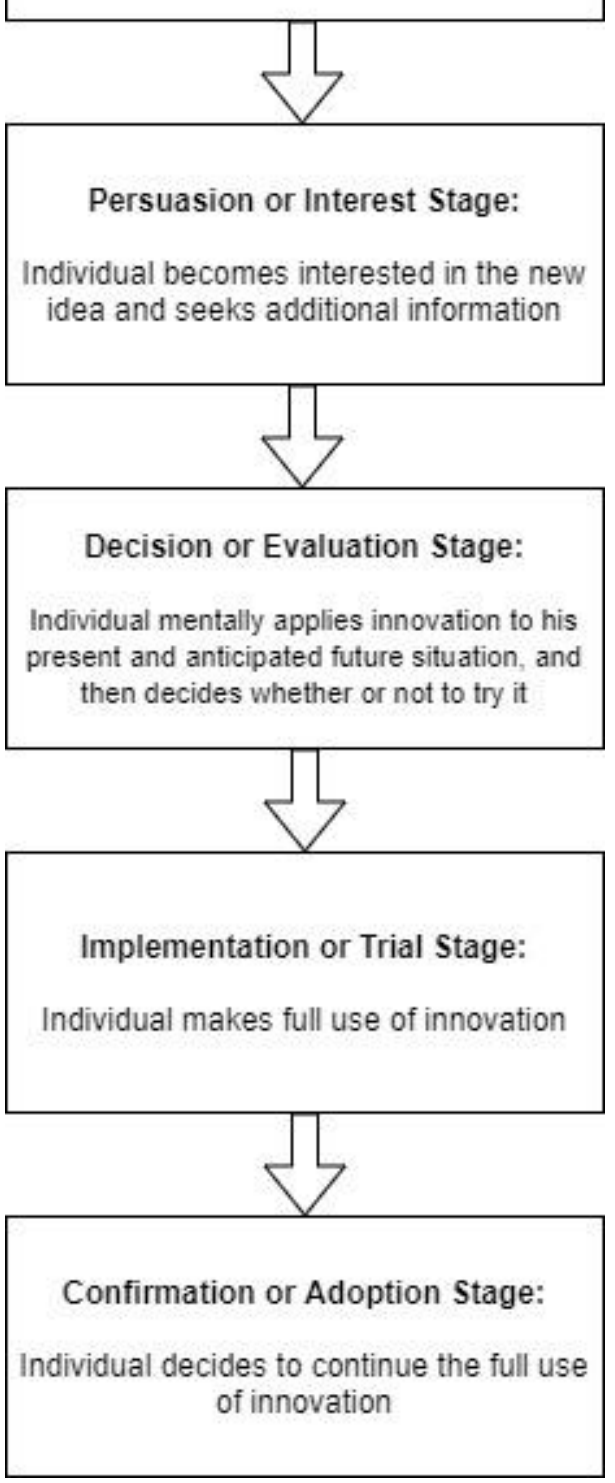

FIGURE 2 INNOVATION ADOPTION PROCESS previous stage the adopter decides whether or not to continue receiving the service from the provider.

One the most important aspects of diffusion of innovation is the consistency of the innovator in following up with the adopter's progress throughout the adoption process. This requires a high level of psychological analysis in order to understand the instant needs and perception of the adopter. 
Using the institutional pressure throughout the adoption process, a proficient innovator should consider adjustments to each of the forces that are subjected to be imposed. For example, if during the third stage of the adoption process, where the adopter compares the available options, the innovator must ensure the competitive advantage over the market in a variety of aspects such as price.

\section{Case study}

Most of the aforementioned are dealing with either lack of interest in embracing a new technology, or utmost, resistance against implementation of a new technology. During a course of a research program previously conducted for developing a new type of concrete chemical admixture $[12,13]$, which utilizes advanced green technologies, a variety of barriers had been experienced. At the beginning, the so-called adopters whom have a long history in the same field, did not catch up with the novelty of the invention. As the mass marketing plans had proceeded, very little attention had been gained by the early adopters. However, during the commercialization process and in the midst of attracting investors, a destructive initiative had faced the program aiming to stop the project to reach its goals. The intention here is for the innovators to become aware of the presence of destructive pressures. Such disturbing forces do actually exist in the built environment. The reason behind these, in some cases, inhumane initiatives is usually the conflict of interest which is driven by competitive type of mentality. The greed to uptake as much resources as possible for personal use is proven to be the dominant mentality behind these type of initiatives. Turning back to the DiMaggio \& Powell's Institutional Theory, it is the institutional isomorphism which satisfies a higher human need as to be the so-called homogeneity. Coalition over competition, in other words, is the fundamental key to success in the modern structuration. Anyways, the approach to tackle such destructive initiatives is still vague.

\section{Conclusion}

The construction industry faces a burden on the environmental initiatives for the last couple of decades, despite the large number of productive and novel technological advancements. It turns out that the resistance against the widespread implementation of these technologies roots from the behavioural characteristics of the individuals and organizations acting in the supply chain. DiMaggio and Powell's institutional theory had been explained in order to define the fundamental behavioural characteristics which lead to organizational success. In combination with Diffusion of Innovation Theory, the innovators in the field of construction had been encouraged to familiarize themselves with the adoption process and at the end a unique case study had been given as an example which still is open for conclusion. 


\section{Reference}

[1] T. Ramesh, R. Prakash, and K. Shukla, "Life cycle energy analysis of buildings: An overview," Energy and buildings, vol. 42, no. 10, pp. 1592-1600, 2010.

[2] A. Todhunter, M. Crowley, M. Gholamisheverini, and F. Sartipi, "Advanced technological implementation of construction and demolition waste recycling," Journal of Construction Materials, vol. 1, no. 1, 2019.

[3] M. Sartipi and F. Sartipi, "Stormwater retention using pervious concrete pavement: Great Western Sydney case study," Case Studies in Construction Materials, vol. 11, p. e00274, 2019.

[4] M. Gamil, A. Ghari Zadeh, and F. Sartipi, "A review on graphene reinforced cement composite: technical approach for ecofriendly construction," Journal of Construction Materials, 2019.

[5] J. Ko, "Carbon: Reducing the Footprint of the Construction Process (London: Construction Products Association) England," in Strategic Forum for Construction and Carbon Trust, 2010.

[6] P. J. DiMaggio and W. W. Powell, "The iron cage revisited: Institutional isomorphism and collective rationality in organizational fields," American sociological review, pp. 147-160, 1983.

[7] H. Muratovic, "Building competitive advantage of the company based on changing organizational culture," Economic Review: Journal of Economics and Business, vol. 11, no. 1, pp. 61-76, 2013.

[8] E. H. Schein, Organizational culture and leadership (Jossey-Bass business \& management series). Jossey Bass Incorporated, 2004.

[9] A. Todhunter, M. Crowley, and F. Sartipi, "Construction productivity indices in socialism compared with capitalism," Journal of Construction Materials, 2019.

[10] J.-J. Tseng and P.-H. Chou, "Mimetic isomorphism and its effect on merger and acquisition activities in Taiwanese financial industries," The Service Industries Journal, vol. 31, no. 9, pp. 1451-1469, 2011.

[11] F. Sartipi, "Organizational structure of construction entities based on the cooperative game theory," Journal of Construction Materials, vol. 1, no. 2, 2020.

[12] A. Todhunter, M. Crowley, F. Sartipi, and K. Jegendran, "Use of the by-products of postcombustion carbon capture in concrete production: Australian case study," Journal of Construction Materials, vol. 1, no. 1, 2019.

[13] A. Gharizadeh, F. Sartipi, E. Ayoubi, and A. Severino, "The chemical reactor design configuration of CO2 concrete green solution," Journal of Construction Materials, vol. 1, pp. 2-5, 2020. 